\title{
Fault Analysis and Automation Technology of 10kv Distribution Line
}

\author{
Jing Hua ${ }^{1, a}$, Yanping Zhou ${ }^{2, b}$, Wei Xiong ${ }^{1, c}$ and Linlin Cao ${ }^{1, d}$ \\ 1, 3, ${ }^{4}$ The Engineering College of Hong he University, Mengzi Yunnan 661199 \\ ${ }^{2}$ Kunming Power Supply Bureau, Kunmin Yunnan 650051 \\ a46951833@qq.com, b76252063@qq.com, 459353359@qq.com, d47976326@qq.com
}

\begin{abstract}
In order to improve the distribution of network fault finding, isolation and restoration line, Firstly, the paper analyzes the current situation of $10 \mathrm{kV}$ distribution lines and the types and characteristics of the fault, The local intelligent processing principle to solve the complex problem of fault isolation and route discovery, In this paper, the realization of distribution network automation technology is analyzed, the principle and characteristics of the system are expounded, and different implementations of different distribution network and fault conditions should be adopted.
\end{abstract}

Keywords: Distribution network; 10kv; Fault; Auto mation; Realization way

\section{$10 \mathrm{kv}$ 配电线路故障分析及其自动化技术浅析}

\author{
华静 $^{1}$ ，周艳平 ${ }^{2}$, 熊伟 ${ }^{1}$, 曹琳琳 $^{1}$
}

（1.红河学院 工学院 云南 蒙自 610000；2. 昆明供电局云南昆明 650051）

摘要: 为了提高配网对网内故障的发现、隔离和线路的复电, 文中首先对国内 $10 \mathrm{kv}$ 配电线路的现状及器故障类型和特点 进行了分析, 提出了采用就地智能处理原则解决对故障的发现、隔离和线路的复电问题, 并针对配网自动化技术的实现方式 进行了分析，阐述了其原理和特点，对于不同的配电网和故障情况应该采用不同的实现方式，具有一定的应用价值。

关键词: 配电网; $10 \mathrm{kv}$, 故障; 自动化; 实现方式。

\section{1. 引言}

馈线自动化是指对配电线路运行状态进行监测和控制, 在故障发生后实现快速准确定位和迅速隔离故 障区段, 恢复非故障区域供电（Feeder Automation, 简称FA）。对于系统中两种类型的输电线路而言, 架 空线路自动化技术实现的主要方式有: 主站集中性、电压电流型、电压时间型、分布智能型等; 而对于电 缆线路，其自动化技术实现方式则只有主站集中性、电压时间型、分布智能型三种。上述的几种自动化技 术均能够快速完成线路故障的定位、隔离、复电。针对架空线路、混合线路和电缆线路的故障停电, 现在 常用的最佳解决原则是就地智能处理原则, 采用该种处理原则, 在技术上具有免维护, 不依赖通信和主站 的优点, 从经济上看, 其具有投资少、见效快、易实施等优点, 就地智能处理原则可分为三种处理策略: 主干分段就地智能处理, 分支分界就地智能处理, 用户分界就地智能处理。

\section{$2.10 \mathrm{kv}$ 配电线路的现状分析}

\section{1 国内 $10 \mathrm{kV}$ 配电线路现状}

在城市核心区, 配电网线路以电缆线路居多, 在一般城区, 城市远郊区及农村又以架空线路居多, 在 核心区与一般城区的交界处则是架空电缆混合线路，架空线路多数是开关本体，基本不具备自动化接口 和改造条件; 电缆线路多数是开关柜，除新装设备，也基本不具备改造条件。

\section{$2.210 \mathrm{kv}$ 配电线路故障类型分析}


要实现配网自动化, 应对各输电线路故障进行正确的分析、隔离和复电, 通过理论及实践总结, 可得 到下述结论：

（1）架空线路故障类型特点

(1)馈线故障出现频繁，会造成大面积停电;

(2)瞬时性故障和单相接地故障多，会造成停电，甚至破坏绝缘，损坏设备;

(3)用户故障不断增加，用户出门故障会造成大面积停电。

（2）电缆线路故障类型特点(1)馈线故障发生概率少，停电次数少;

(2)永久性故障占多数，会造成大面积停电;

(3)故障点更多分布在配电站点内和电缆接头处, 属于发展性故障, 会破坏绝缘甚至损伤设备;

(4)用户故障不断增加用户出门故障会造成大面积停电。

（3）架空线路和架空电缆混合线路的故障停电问题则是配网自动化就地智能处理重点解决的问题。

\section{3. 馈线自动化实现方式}

结合 $10 \mathrm{kv}$ 配电网的故障类型和特点, 为提高配电网发现、隔离、恢复故障的能力, 实现配电网自动化, 现阶段主要的配网自动化实现方式如下:

\section{1 主站集中型 FA 技术}

1、工作原理

主站系统根据配电终端检测到的故障告警信息, 结合变电站的保护动作信号进行综合判断, 确定故障 类型和故障区段, 自动或手动隔离故障点, 恢复非故障区段的供电。

2、特点

（1）线路类型无限制，架空、电缆、混合都适合;

(2) 不要求变电站重合闸配合

（3）无须保护配合，实施方便，管理简单

(4) 分段不受限, 扩展灵活

（5）主干线路开关采用蓄电池作为后备电源，分界开关配置超级电容作为后备电源

（6）依赖通信及主站，投资较大，每次故障，整条线路存在短时停电。

\section{2 分布智能型 FA 技术}

\section{1、工作原理}

配电馈线主干线上各个相邻开关的配电终端通过通信网络（光纤/GPRS），相互交换电压/过流、开关 位置和故障状态等多种信息。发生故障时不需主站参与控制快速隔离; 而后通过通信获得相关的开关已经 隔离故障信息，按预定的恢复机制恢复正常区间供电的一种馈线自动化系统。

2、特点

（1）DTU可自行收集处理相关故障信息并进行控制决策

(2) 不会对系统造成多次过流冲击

（3）架空与电缆线路都适用

（4）不依赖于主站完成配电网故障自愈控制, 动作速度快 
(5) 对通信的速度、稳定、要求比较高; 投资比较大运维要求高

\section{3 电压时间型 FA 技术}

1、工作原理

主干线分段及联络开关采用 “电压一时间型”负荷开关，与变电站重合闸配合，自动隔离故障，恢复 非故障区间的供电。当线路发生短路故障时, 变电站保护跳闸, 第一次重合闸, 开关得电后逐级延时合闸, 当合闸到故障点后, 变电站再次跳闸, 同时FTU通过电压一时间逻辑判断出故障点并闭锁两端开关; 故障 隔离后, 变电站二次重合, 恢复故障点前段线路供电, 联络开关延时合闸, 自动恢复故障点后段线路供电。 2、特点

(1)开关采用 “来电即合、无压释放” 的原理, 无蓄电池, 真正免维护;

(2)不依赖通信及主站实现就地智能保护功能;

(3)资金投入小，周期短，见效快;

(4)适合城郊或者农村架空中压配电线路;

(5)需要变电站2次重合闸配合, 用户须受到2次短时停送电影响;

(6)故障区段负荷侧恢复供电时间大于 $45 \mathrm{~S}$ 。

\section{4 电压电流型 FA 技术}

\section{1、工作原理}

电压电流型馈线自动化是在电压-时间型基础上，增加了故障电流辅助判据。使非故障区间分段开关在 第一次重合闸后闭锁分闸，减少第二次重合闸后恢复供电时间。其次主干线设置带时限和二次重合闸的 分段断路器, 其后端线路发生故障自行切除, 大大减少变电站出线断路器的跳闸次数。

2、特点

(1) 减少 $50 \%$ 变电站出线断路器跳闸, 缩小故障引起的停电范围;

（2）变电站出线断路器重合成功率大幅提高, 可达到 $90 \%$ 以上;

（3）减少重合闸恢复供电时逐级合闸时间，减少非故障区段停电时间;

（4）无通信可实现故障的就地迅速隔离;

(5) 适用于 $10 \mathrm{kV}$ 架空、电缆等典型结线;

(6) 要求变电站保护动作时间至少是在 $0.15 \mathrm{~S}$ 以上。

\section{4. 结语}

将主站系统接入升级线路中, 采用FA就地智能故障处理方可在输电线路中实现对线路运行状态的管、 监、控、优, 随着电力系统的不断发展, 配电网结构越来越复杂, 配网中某条线路的小故障若不及时切除 将会影响到其他线路, 甚至演变成为三相短路, 扩大故障的影响范围, 实现配网自动化将能够有效解决上 述问题。

\section{5. 致谢}

基金项目：云南省教育厅科学研究基金资助项目（2015Y453）

\section{Acknowledgment}


Fund Project: Yunnan Provincial Department of Education Science Research Fund Project (2015Y453)

\section{参考文献}

[1] 张保会, 尹项根. 电力系统继电保护 [M]. 北京: 中国电力出版社, 2005

[2] 王士政. 电网调度自动化与配网自动化技术 [M] . 北京: 水利水电出版社, 2006.

[3] 韩国政, 徐丙垠, 等. 基于IEC61850的配网自动化通信技术研究 [J] 电力系统保护与控制, 2013（2)：62-66.

[4] 范颖, 高新华. 广州供电局配网自动化系统的新技术应用及研究 $[\mathrm{J}]$ 南方电网技术, 2009, 3（5): 64-68.

[5]阎阳.配网自动化通信技术的研究 [J]华北电力大学, 2011.

[6] 刘红松. 配网自动化建设与运行管理问题研究 [J] 中国电力教育, 2011,20 (20): 97-98.

[7] 梁汉泉. 配网自动化及通信系统的规划建设 $[\mathrm{J}]$ 中国科技投资，2013（A25:148-148）

\section{References}

[1] Zhang Baohui, Yin Xianggen. Power system relay protection [M]. Beijing: China Electric Power Press, 2005

[2] Wang Shizheng. Power grid dispatching automation and distribution network automation technology [M]. Beijing: Water Resources and Hydropower Press, 2006.

[3] Han Guozheng, Xu Bingyin, etc. Based on IEC61850 distribution network automation communication technology research [J]. power system protection and control, 2013 (2): 62-66.

[4] Fan Ying, Gao Xinhua. New technology applications and research of Guangzhou Power Supply Bureau distribution network automation system [J]. Southern Power Grid Technology, 2009, 3 (5): 64-68.

[5] Yan Yang. Distribution network automation communication technology research [J]. North China Electric Power University, 2011.

[6] Liu Hongsong. Study on distribution network automation construction and issues of operation and management [J]. China Electric Power Education, 2011, 20 (20): 97-98.

[7] Liang Hanquan. Distribution network automation and planning construction of communication system [J] China Science and Technology Investment, 2013 (A25: 148-148) 